\title{
ХВИЛЕВІДНИЙ ДИФЕРЕНЦІЙНИЙ ФАЗОЗСУВАЧ НА 90 У ДІАПАЗОНІ ЧАСТОТ 7-10 ГГЦ
}

\author{
Національний технічний університет України «Київський політехнічний інститут ім. Ігоря \\ Сікорського»;
}

\begin{abstract}
Анотація
Запропоновано результати дослідження та розробки хвилеводного диференційного фазозсувача у діапазоні частот 7-10 ГГи.

Ключові слова: фазозсувач, хвилевід, діафрагма, диференційний фазовий зсув, коефіцієнт відбиття.
\end{abstract}

\begin{abstract}
The results of research and development of waveguide differential phase shifter in the frequency range 7-10 GHz are proposed.

Keywords: phase shifter, waveguide, iris, differential phase shift, reflection coefficient.

\section{Ветуп}

Сучасні антенні системи із поляризаційною обробкою сигналів використовують для покращення інформаційних параметрів сигналів, що в них обробляються [1-4]. Базовими елементами таких систем $є$ фазозсувачі та поляризатори. Фазозсувачі забезпечують на виході необхідні значення фази [5-9]. Поляризатори забезпечують на виході диференційний фазовий зсув в 90 [10-16]. Найбільш поширеними структурами хвилеводних поляризаторів є структури із діафрагмами [17-25], штирями [26-30], комбіновані [31-33], та коаксіальними і ребристими структурами [34-45]. Диференційний фазозсувач використовується при розробці фазованих антенних решіток, при формуванні променя в сучасних антенних системах та для систем живлення сучасних антен [46-49]. Сучасні фазозсувачі на $90^{\circ}$ [50-53] має розширені функціональні можливості, просту компактну геометричну форму, стійкий до амплітудного розбалансування, можливість підтримання стабільною фазу при багатопроменевому поширенню. Крім того, такі пристрої іноді використовуються в системах 5G [54-72].
\end{abstract}

Метою роботи є дослідження основних характеристик диференційного фазозсувача діапазону частот 7-10 ГГц.

\section{Результати дослідження}

Конструктивно диференційного хвилеводного фазозсувача з трьома діафрагмами, що розміщені симетрично, поданий на рис. 1 . Висота двох крайніх діафрагм рівна $h_{1}$, а висота середньої вища за крайні $h_{2}$, товщина всіх діафрагм $w$, та відстань між діафрагмами $є$ однакова і становить $l$.

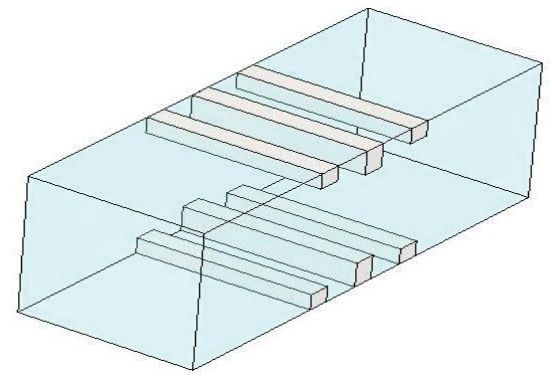

Рис. 1. Конструкція поляризатора

Розроблена конструкція фазозсувного пристрою забезпечує основні фазові та узгоджувальні характеристики. Оптимізація конструкції здійснюється у відповідному комерційному програмноу забезпеченню за рахунок зміни геометричних розмірів конструкції. 
За допомогою методу хвильових матриць були отримані основних параметри хвильової матриці розсіювання фазозсувача:

$$
\left[S_{\Sigma}\right]=\left[\begin{array}{ll}
S_{11 . \Sigma} & S_{12 . \Sigma} \\
S_{21 . \Sigma} & S_{22 . \Sigma}
\end{array}\right]
$$

Таким чино через елементи отриманої матриці були визначений диференційний фазовий зсув пристрою відповідно до формули:

$$
\Delta \varphi=\varphi_{21 . \Sigma \mathrm{L}}-\varphi_{21 . \Sigma \mathrm{C}}
$$

Нижче подані графічні характеристики запропонованого хвилеводного фазозсувного пристрою на основі трьох діафрагм у робочому діапазоні частот 7-0 ГГц для поданого методу та двох відомих електродинамічних методів FDTD та FEM відповідно.

Рис. 2 містить залежність диференційного фазового зсуву хвилеводного фазозсувача у діапазоні частот 7-10 ГГц. Як видно, диференційний фазовий зсув пристрою $є 90^{\circ} \pm 5^{\circ}$ для поданого методу, $90^{\circ} \pm 4^{\circ}$ для методу FDTD та $90^{\circ} \pm 6^{\circ}$ для методу FEM.

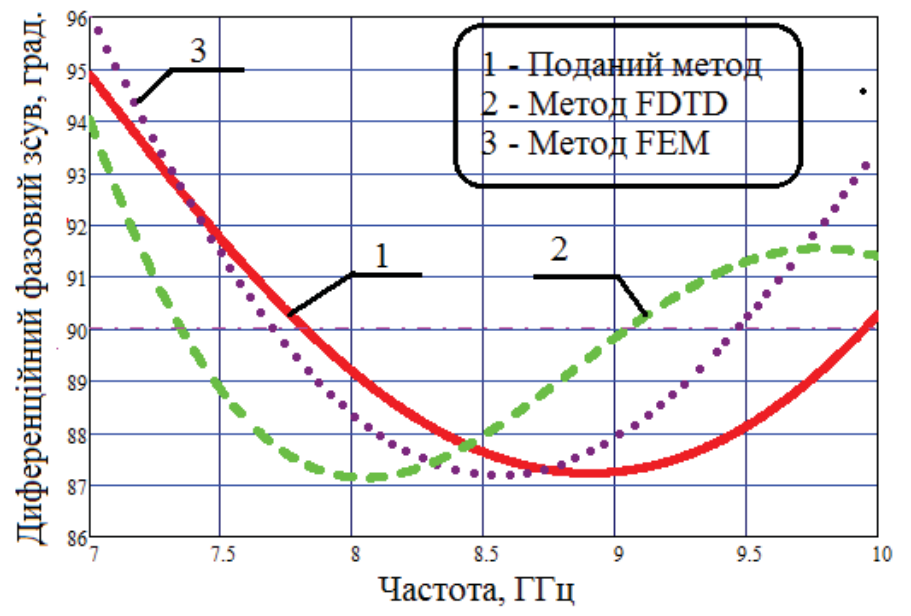

Рис. 3. Залежність диференційного фазового зсуву від частоти

На рис. 3 представлена графічна залежність коефіцієнта відбиття розробленого фазозсувного пристрою для горизонтальної та вертикальної поляризації для поданого методу, методу FDTD, методу FEM. Суцільною лінією зображена вертикальна поляризація, а точковими подана горизонтальна поляризація. Як видно, максимальний рівень коефіцієнта відбиття є 0.6 для поданого методу, 0,52 для методу FDTD та 0.63 для методу FEM.

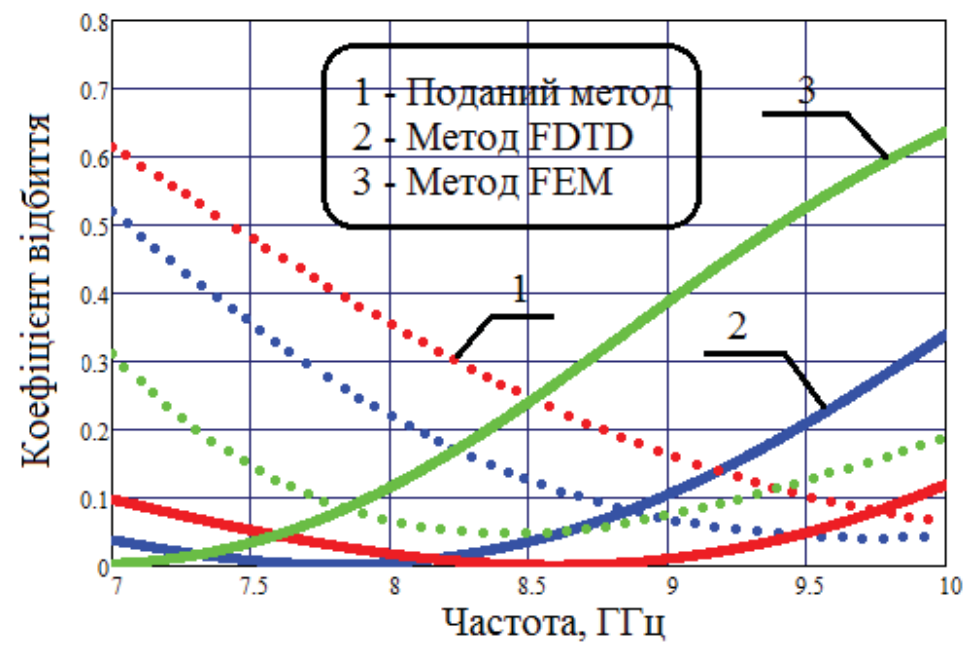

Рис. 2. Залежність коефіцієнта відбиття від частоти для обох поляризацій

Таким чином, результати проектування пристрою поданим методом та відомими електродинамічними методами корелюють між собою [73-80]. 


\section{Висновки}

В дослідженні був розроблений диференційний фазо зсувний пристрій на основі трьох діафрагм у робочому діапазоні 7-10 ГГЦ. Представлений пристрій підтримує диференційний фазовий зсув $90^{\circ} \pm 5^{\circ}$. Пікове значення коефіцієнту відбиття для вертикальної та горизонтальної поляризацій становить 0,52 на частоті 7 ГГц. Отже, запропонований пристрій може використовуватися у сучасних фазованих антенних решітках.

\section{СПИСОК ВИКОРИСТАНОЇ ЛІТЕРАТУРИ}

1. Stutzman W.L. Polarization in Electromagnetic Systems, Artech House, Norwood 2018, 352 p.

2. Piltyay S. Information resources economy in satellite systems based on new microwave polarizers with tunable posts / S. Piltyay, A. Bulashenko, H. Kushnir, O. Bulashenko // Path of Science. - 2020. - Vol. 6, No 11. - pp. 5001-5010. http://doi.org/10.22178/pos.55-1.

3. Hwand S. Design of a full-band polariser used in WR-22 standard waveguide for satellite communications / S. Hwand, K. Lee // The Journal of Engineering. - 2014. - Vol. 9. - pp. 508-513. DOI: 10.1049/JOE.2014.0216.

4. Bulashenko A.V. Wave matrix technique for waveguide iris polarizers simulation. Theory / A.V. Bulashenko, S.I. Piltyay, I.V. Demchenko // Journal of Nano- and Electronic Physics. - 2020. - Vol. 12, no. 6. - pp. 06026-1-06026-5. DOI: 10.21272/jnep.12(6).06026.

5. Sheikh S.I.M., Gibson A.A.P., Basorrah M. Time-delay phase shifters for mmWave phase arrays / S.I.M.. Sheikh, A.A.P. Gibson, M. Basorrah, G. Alhulwah, K. Alanizi, M. Alfarsi, J. Zafar // IEEE Antennas and Wireless Propagation Letters. - 2010. - Vol. 9. - p. 319-321. DOI: 10.1109/LAWP.2010.2048190.

6. Lee H.-S. W-band CMOS 4-bit shift for high power and phase compression points / H.-S. Lee, B.W. Min // IEEE Transactions on Circuits and Systems II: Express Briefs. - 2015. - Vol. 62, no. 1. - p. 1-5. DOI: 10.1109/TCSII.2014.2362732.

7. Singh T. Time-delay phase shifters for mmWave phase arrays / T. Singh, R.R. Mansour // IEEE Transactions on Microwave Theory and Techniques. - 2020. - Vol. 68, no. 9. - p. 3745-3755. DOI: 10.1109/TMTT.2020.3007833

8. Amin F. Compact and low-loss phase shifters and multibit phase shifters based on inverted-E topology / F. Amin, Y. Liu, Y. Zhao, S. Hu // IEEE Transactions on Microwave Theory and Techniques. - 2021. Vol. 69, No. 4. - pp. 2120-2129. DOI: 10.1109/TMTT.2021.3061482.

9. Hensley D.M. A stretchable liquid metal coaxial phase shifter / D.M. Hensley, C.G. Christodoulou, N. Jackson // IEEE Open Journal of Antennas and Propagation. - 2021. - Vol. 2. - pp. 370-374. DOI: 10.1109/OJAP.2021.3063289.

10. Virone G. Optimum-iris-set concept for waveguide polarizers / G. Virone, R. Tascone, O.A. Peverini and R. Orta // IEEE Microwave and Wireless Components Letters. - 2007. - Vol. 17, no. 3. p. 202-204. DOI: 10.1109/LMWC.2006.890474.

11. Arndt F. Design of a wideband compact square waveguide polarizer / F. Arndt, W. Tuchkone, T. Wriedt // Electronics Letters. - 1985. - Vol. 21, №12. - p. 517-518. DOI: 10.1049/el:19850365.

12. Piltyay S.I. Compact Ku-band iris polarizers for satellite telecommunication systems / S.I. Piltyay, O.Yu. Sushko, A.V. Bulashenko, I.V. Demchenko // Telecommunications and Radio Engineering. - 2020. Vol. 79, no. 19. - pp. 1673-1690. DOI:10.1615/TelecomRadEng.v79.i19.10.

13. Bulashenko A.V. Wave matrix technique for waveguide iris polarizers simulation. Theory / A.V. Bulashenko, S.I. Piltyay, I.V. Demchenko // Journal of Nano- and Electronic Physics. - 2020. - Vol. 12, no. 6. - pp. 06026-1-06026-5. DOI: 10.21272/jnep.12(6).06026.

14. Bulashenko A.V. Equivalent microwave circuit technique for waveguide iris polarizers development / A.V. Bulashenko, S.I. Piltyay // Visnyk NTUU KPI Seriia - Radiotekhnika, Radioaparatobuduvannia. 2020. - Vol. 83. - pp. 17-28. http://doi.org/10.20535/RADAP.2020.83.17-28.

15. Piltyay S.I. Analytical synthesis of waveguide iris polarizers / S.I. Piltyay, A.V. Bulashenko, I.V. Demchenko // Telecommunications and Radio Engineering. - 2020. - Vol. 79, No 18. - pp. 1579-1597. http://doi.org/10.1615/TelecomRadEng.v79.i18.10. 
16. Bulashenko A.V. Optimization of a polarizer based on a square waveguide with irises / A.V. Bulashenko, S.I. Piltyay, I.V. Demchenko // Science-Based Technologies. - 2020. - Vol. 47, No. 3. - pp. 287-297. (in Ukrainian). http://doi.org/10.18372/2310-5461.47.14878.

17. Bulashenko A.V. Waveguide polarizer with three irises for antennas of satellite television systems / A.V. Bulashenko, S.I. Piltyay, H.S. Kushnir, O.V. Bulashenko // Science-Based Technologies. - 2020. Vol. 49, No. 1. - pp. 39-48. (in Ukrainian). http://doi.org/10.18372/2310-5461.49.15290.

18. Piltyay S. FDTD and FEM simulation of microwave waveguide polarizers / S. Piltyay, A.Bulashenko, Ye. Herhil, O. Bulashenko // IEEE 2nd Int. Conf. on Advanced Trends in Information Theory, 25-27 November 2020, Kyiv, Ukraine, pp. 132-137. DOI: 10.1109/ATIT50783.2020.9349339.

19. Bulashenko A.V. Analytical technique for iris polarizers development / A.V. Bulashenko, S.I. Piltay, I.V. Demchenko // IEEE International Conference on Problems of Infocommunications. Science and Technology (PIC S\&T). - Kharkiv, Ukraine, 2020. - pp. 471-476.

20. Piltyay S.I. Compact polarizers for satellite information systems / S.I. Piltyay, A.V. Bulashenko, I.V. Demchenko // IEEE International Conference on Problems of Infocommunications. Science and Technology, 8-10 October 2020, Kharkiv, Ukraine, pp. 350-355.

21. Piltyay S.I. Waveguide iris polarizers for Ku-band satellite antenna feeds / S.I. Piltyay, A.V. Bulashenko, I.V. Demchenko // Journal of Nano- and Electronic Physics. - 2020. - Vol. 12, No. 5. pp. 05024-1-05024-5. http://doi.org/10.21272/jnep.12(5).05024.

22. Piltyay S.I. Numerical performance of FEM and FDTD methods for the simulation of waveguide polarizers / S.I. Piltyay, A.V. Bulashenko, Y.Y. Herhil // Visnik NTUU KPI Seriia - Radiotekhnika, Radioaparatobuduvannia. - 2021. - Vol. 84. - pp. 11-21. DOI:10.20535/RADAP.2021.84.11-21.

23.Piltyay S.I. High performance waveguide polarizer for satellite information systems / S.I. Piltyay, A.V. Bulashenko, Ye.I. Kalinichenko, O.V. Bulashenko // Bulletin of Cherkasy State Technological University. - 2020. - Vol. 4. - pp. 14-26. [In Ukrainian]. DOI: 10.24025/2306-4412.4.2020.217129.

24. Bulashenko A.V. Simulation of compact polarizers for satellite telecommunication systems with the account of irises' thickness / A.V. Bulashenko, S.I. Piltyay, I.V. Demchenko // KPI Science news. - 2021. Vol. 1. - pp. 25-33. http://doi.org/10.20535/kpi-sn.2021.1.203863.

25. Булашенко А.В. Конструкція портативного цифрового мегомметра та вимірювача струму витоку / А.В. Булашенко, І.В. Забегалов // Вісник ВПІ. - 2020. - Вип. 3. - с. 37-42. https://doi.org/10.31649/1997-9266-2020-150-3-37-42.

26.Ruiz-Cruz A. Compact reconfigurable waveguide circular polarizers / A. Ruiz-Cruz, M. M. Fahmi, M. Daneshmand, and R. R. Mansour // IEEE Microwave Symposium Digest, Baltimore, USA, June 2011, pp. 1-4. DOI: 10.1109/MWSYM.2011.5972872.

27.Chittora A. A compact circular waveguide polarizer with higher order mode excitation / A. Chittora, S.V. Yadav // IEEE International Conference on Electronics, Computing and Communication Technologies, Bangalore, India, July 2020. DOI: 10.1109/CONECCT50063.2020.9198499.

28.Subbarao B. Compact coaxial-fed polarizer / B. Subbarao, V.F. Fusco // IEEE Antennas and Wireless Propagation Letters. - 2004. - Vol. 3. - pp. 145-147. DOI: 10.1109/LAWP.2004.831084.

29.Piltyay S. Information resources economy in satellite systems based on new microwave polarizers with tunable posts / S. Piltyay, A. Bulashenko, H. Kushnir, O. Bulashenko // Path of Science. - 2020. - Vol. 6, No 11. - pp. 5001-5010. http://doi.org/10.22178/pos.55-1

30. Bulashenko A.V. Compact waveguide polarizer with three antiphase posts / A.V. Bulashenko, S.I. Piltyay, H.S. Kushnir, O.V. Bulashenko // Visnyk VPI. - 2020. - Vol. 5. - pp. 97-104. [In Ukrainian]. DOI: 10.31649/1997-9266-2020-151-5-97-104.

31. Bulashenko A.V. Tunable square waveguide polarizer with irises and posts / A.V. Bulashenko, S.I. Piltyay, Y.I. Kalinichenko, O.V. Bulashenko // Technical Engineering. - 2020. - Vol. 86, no 2. - pp. 108116. [In Ukrainian]. DOI: 10.26642/ten-2020-2(86)-108-116.

32. Piltyay S. New tunable iris-post square waveguide polarizers for satellite information systems / S. Piltyay, A. Bulashenko, H. Kushnir, O. Bulashenko // IEEE 2nd International Conference on Advanced Trends in Information Theory, 25-27 November 2020, Kyiv, Ukraine, pp. 342-348. DOI: 10.1109/ATIT50783.2020.9349357.

33. Bulashenko A. Mathematical modeling of iris-post sections for waveguide filters, phase shifters and polarizers / A. Bulashenko, S. Piltyay, Ye. Kalinichenko, O. Bulashenko // IEEE 2nd International 
Conference on Advanced Trends in Information Theory, 25-27 November 2020, Kyiv, Ukraine, pp. 330-336. DOI: 10.1109/ATIT50783.2020.9349321.

34. Dubrovka F. Boundary problem solution for eigenmodes in coaxial quad-ridged waveguides / F. Dubrovka, S. Piltyay // Information and Telecommunication Science. - 2014. - Vol. 5, no. 1. - pp. 48-61. DOI: 10.20535/2411-2976.12014.48-61.

35. Dubrovka F. Prediction of eigenmodes cutoff frequencies of sectoral coaxial ridged waveguides / F. Dubrovka, S. Piltyay // IEEE International Conference on Modern Problem of Radio Engineering, Telecommunications and Computer Science, 21-24 February 2012, Lviv, Ukraine.

36. Naydenko V. Evolution of radiopulses radiated by Hertz's dipole in vacuum / V. Naydenko, S. Piltyay // IEEE International Conference on Mathematical Methods in Electromagnetic, 1-2 July 2008, Odessa, Ukraine. DOI: 10.1109/MMET.2008.4580972.

37. Dubrovka F.F. Eigenmodes analysis of sectoral coaxial ridged waveguides by transverse fieldmathing technique. Part 1. Theory. / F.F. Dubrovka, S.I. Piltyay // Visnyk NTUU KPI Seriia Radiotekhnika, Radioaparatobuduvannia. - 2013. - Vol. 54. - pp. 13-23. http://doi.org/10.20535/RADAP.2013.54.13-23.

38. Dubrovka F. F. Eigenmodes of sectoral coaxial ridged waveguides // F.F. Dubrovka, S.I. Piltyay // Radioelectronics and Communications Systems. - 2012. - Vol. 55, № 6. - P. 239-247. DOI: https://doi.org/10.3103/S0735272712060015.

39. Dubrovka F. F. Eigenmodes of coaxial quad-ridged waveguides. Numerical results // F.F. Dubrovka, S.I. Piltyay // Radioelectronics and Communications Systems. — 2014. — Vol. 57, № 2. — P. 59-69. DOI: https://doi.org/10.3103/S0735272714020010.

40. Dubrovka F. A novel wideband coaxial polarizer / F. Dubrovka, S. Piltyay // IX International Conference on Antenna Theory and Techniques, 16-20 Sept. 2013, Odessa, Ukraine, pp. 473-474. DOI: 10.1109/ICATT.2013.6650816.

41. Dubrovka F. Numerically effective basis functions in integral equation technique for sectoral coaxial ridged waveguides / F. Dubrovka, S. Piltyay // XI International Conference on Mathematical Methods in Electromagnetic Theory, 28-30 Aug. 2012, Kharkiv, Ukraine, pp. 492-495. DOI: 10.1109/MMET.2012.6331195.

42. Piltyay S.I. Enhanced C-band coaxial orthomode transducer / S.I. Piltyay // Visnyk NTUU KPI Seriia - Radiotekhnika, Radioaparatobuduvannia. - 2014. - Vol. 58. - pp. 27-34. http://doi.org/10.20535/RADAP.2014.58.27-34.

43. Dubrovka F. A high performance ultrawideband orthomode transducer and dual-polarized quadridged horn antenna based on it / F. Dubrovka, S. Piltyay // IEEE International Conference on Antenna Theory and Techniques, 20-23 Sept. 2011, Lviv, Ukraine, pp. 176-178. DOI: 10.1109/ICATT.2011.6170737.

44. Dubrovka F. Novel high performance choherent dual-wideband orthomode transducer for coaxial horn feeds / F. Dubrovka, S. Piltyay // XI International Conference on Antenna Theory and Techniques, 2427 May 2017, Kyiv, Ukraine, pp. 277-280. DOI: 10.1109/ICATT.2017.7972642.

45.Bulashenko A.V. Wave matrix technique for waveguide iris polarizers simulation. Theory / A.V. Bulashenko, S.I. Piltyay, I.V. Demchenko // Journal of Nano- and Electronic Physics. - 2020. - Vol. 12, no. 6. - pp. 06026-1-06026-5. DOI: 10.21272/jnep.12(6).06026.

46. Bulashenko A.V. Beamforming principels of smart antennas / A.V. Bulashenko // Visnik Sumy State University. Seriia Technical sciences. - 2010. - Vol. 1. - pp. 111-120.

47. Булашенко А.В. Живлення антенних решіток на основі лінз Ротмана (огляд) / А.В. Булашенко, Ф.Ф. Дубровка // Вісник Сумського державного університету. Серія Технічні науки. - 2010. - №3, Т.2. - C. 113-120.

48. Bulashenko A.V. Multibeam arrays on the basis of Rotman lenses / A.V. Bulashenko // Visnyk NTUU KPI Seriia - Radiotekhnika, Radioaparatobuduvannia. - 2010. - Vol. 42. - pp. 178-186. http://doi.org/10.20535/RADAP.2010.42.178-186.

49. Sushko O. Symmetrically fed 1-10 GHz log-periodic dipole antenna array feed for reflector antennas / O. Sushko, S. Piltyay, F. Dubrovka, // IEEE Ukraine Microwave Week, 21-25 Sept. 2020, Kharkiv, Ukraine. DOI: 10.1109/UkrMW49653.2020.9252778.

50. Hensley D.M. Astretchable liquid metal coaxial phase shifter / D.M. Hensley, C.G. Christodoulou, N. Jackson // IEEE Open Journal of Antennas . - 2021. - Vol. 2. - pp. 370-374. DOI:10.1109/OJAP.2021.3063289. 
51. Amin F. Compact and low-loss phase shifters and multibit phase shifters based on inverted-E topology / F. Amin, Y. Liu, Y. Zhao, S. Hu // IEEE Transactions on Microwave Theory and Techniques . - 2021. - Vol. 69, No. 4. - pp. 2120-2129. DOI:10.1109/TMTT.2021.3061482.

52. Wu T. A $60-\mathrm{GHz}$ variable gain phasr shifter with 14.8-dB gain tuning range and 6-bit phase resolution across $-25^{\circ} \mathrm{C}-110^{\circ} \mathrm{C} / \mathrm{T}$. Wu, Y. Wu, X. Zhang, Z. Xing, C. Zhao, H. Liu, Y. Yu, K. Kang // IEEE Transactions on Microwave Theory and Techniques. - 2021. - Vol. 69, No. 4. - pp. 2371-2385. 2021. DOI:10.1109/TMTT.2021.3057390.

53. Huang C. Photonic-assisted microwave frequency and phase shifter for deception jamming / C. Huang, E.H.W. Chan // IEEE Photonics Journal. - 2021. - Vol. 13, No. 3. DOI:10.1109/JPHOT.2021.3074164.

54. Bulashenko A.V. Energy efficiency of the D2D direct connection system in 5G networks / A.V. Bulashenko, S.I. Piltyay, I.V. Demchenko // IEEE International Conference on Problems of Infocommunications. Science and Technology, 8-10 October 2020, Kharkiv, Ukraine, pp. 324-329.

55.Piltyay S.I. Wireless sensor network connectivity in heterogeneous 5G mobile systems / S.I. Piltyay, A.V. Bulashenko, I.V. Demchenko // IEEE International Conference on Problems of Infocommunications. Science and Technology (PIC S\&T), 8-10 October 2020, Kharkiv, Ukraine, pp. 508-513.

56.Bulashenko A.V. Combined criterion for the choice of routing based on D2D technology / A.V. Bulashenko // Radio Electronics, Computer Science, Control. - 2021. - Vol. 1. - pp. 7-13. (in Ukrainian). http://doi.org/10.15588/1607-3274-2021-1-1.

57.Bulashenko A.V. Evaluation of D2D Communications in 5G networks / A.V. Bulashenko // Visnyk NTUU KPI Seriia - Radiotekhnika, Radioaparatobuduvannia. - 2020. - Vol. 81. - pp. 21-29. (in Ukrainian). http://doi.org/10.20535/RADAP.2020.81.21-29.

58. Bulashenko A.V. Data upload system using D2D technology in the unlicensed frequency range as part of the 5G communication system / A.V. Bulashenko // Technical Engineering. - 2020. - Vol. 86, No. 2. pp. 103-107. (in Ukrainian). http://doi.org/10.26642/ten-2020-2(86)-103-107.

59.Barki A. M2M security: challenges and solutions / A. Barki, A. Bouabdallah, S. Gharout, Y. Traore // IEEE Communications Surveys \& Tutorials. - 2016. - Vol. 18, No. 2. - pp. 1241-1254. DOI: 10.1109/COMST.2016.2515516.

60. Bulashenko A. New traffic model of M2M Technology in 5G wireless sensor networks / A. Bulashenko, S. Piltyay, A. Polishchuk, O. Bulashenko // IEEE 2nd International Conference on Advanced Trends in Information Theory, 25-27 November 2020, Kyiv, Ukraine, pp. 125-131. http://doi.org/10.1109/ATIT50783.2020.9349305.

61. Bulashenko A.V. Resource allocation for low-power devices of M2M technology in 5G networks / A.V. Bulashenko // KPI Science news. - 2020. - Vol. 3. - pp. 7-13. (In Ukrainian). http://doi.org/10.20535/kpi-sn.2020.3.203863.

62. Myronchuk O. Two-stage channel frequency response estimation in OFDM systems / O. Myronchuk, O. Shpylka, S. Zhuk // Path of Science. - 2020. - Vol. 6, No. 2. - pp. 1001-1007. DOI: 10.22178/pos.55-1.

63. Myronchuk O. Algorithm of channel frequency response estimation in orthogonal frequency division multiplexing systems based on Kalman filter /O. Myronchuk, O. Shpylka, S. Zhuk // IEEE 15th International Conference on Advanced Trends in Radioelectronics, Telecommunications and Computer Engineering, 2529 Feb. 2020, Lviv-Slavske, Ukraine. DOI:10.1109/TCSET49122.2020.235385.

64. Myronchuk O.Y. Two-Stage Method for Joint Estimation of Information Symbols and Channel Frequency Response in OFDM Communication Systems / O.Y. Myronchuk, A.A. Shpylka, S.Y. Zhuk // Radioelectronics Communications System. - 2020. - Vol.63. - pp. 418-429. https://doi.org/10.3103/S073527272008004X.

65. Myronchuk A.Y. Channel frequency response estimation method based on pilot's filtration and extrapolation / A.Y. Myronchuk, O.O. Shpylka, S.Y. Zhuk // Visnyk NTUU KPI Seriia - Radiotekhnika Radioaparatobuduvannia. - 2019. - Vol. 78. - pp. 36-42. DOI: 10.20535/RADAP.2019.78.36-42.

66. Гнитецький В.А. Забезпечення дуже низьких затримок у стільниковій системі 5G на базі МЕC / В.А. Гнитецький, А.В. Булашенко // Міжнародна науково-технічна конференція «Радіотехнічні поля, сигнали, апарати та системи». Київ, 18 - 24 листопада 2019 р - Київ, 2019. - С. 153 - 155.

67. Гладун В.В. Забезпечення високої якості мережі $5 \mathrm{G}$ за допомогою технології D2D / B.B. Гладун, А.В. Булашенко // Міжнародна науково-технічна конференція «Радіотехнічні поля, сигнали, апарати та системи». Київ, 18 - 24 листопада 2019 р - Київ, 2019. - С. 57 - 59. 
68. Ляшко Д.Г. Спільне використання ресурсів D2D та мобільного зв'язку / Д.Г. Ляшко, А.В. Булашенко // Матеріали V Всеукраїнської науково-методичної конференції, м. Шостка, 23 квітня 2020 року. - Суми: Сумський державний університет, 2020. - С. 170 -171.

69. Булашенко A.B. Побудова векторних діаграм за допомогою математичного пакету MathCAD / А.В. Булашенко // Науково-методична конференція викладачів, співробітників і студентів: тези доповідей, 27 квітня 2010 року. - Суми : СумДУ, 2010. - Ч.2. - С. 10-13.

70. Гладун B.B. Система вигризки на основі D2D / B.В. Гладун, А.В. Булашенко // Матеріали V Всеукраїнської науково-методичної конференції, м. Шостка, 23 квітня 2020 року. - Суми: Сумський державний університет, 2020. - С. $166-167$.

71. Булашенко А.В. Аналіз можливостей хмарних технологій / А.В. Булашенко, О.Л. Литвинець // Матеріали II Всеукраїнської науково-методичної конференції, м. Шостка, 20 квітня 2017 року. Суми: Сумський державний університет, 2020.

72.Zhuk S.Ya. Synthesis of extremely wide stopband E-plane bandpass filters / S.Ya. Zhuk, M.Y. Omelianenko, T.M. Romanenko, O.V. Tureeva // Visnyk NTUU KPI Seriia - Radiotekhnika, Radioaparatobuduvannia. - 2021. - Vol. 84. - pp. 22-29. http://doi.org/10.20535/RADAP.2021.84.22-29.

73. Omelianenko M.Yu. Stopband characteristics improvement of waveguide planar E-plane filters // M.Yu. Omelianenko, T.M. Romanenko, S.Ya. Zhuk, O.V. Turieieva // Radioelectronics and Communications Systems. - 2021. - Vol. 64, № 2. - P. 53-63. DOI: https://doi.org/10.3103/S0735272721020011.

74.Tovakch I.O. Adaptive filtration of parameters of the UAV movement on data from its location on the basis the time difference of arrival method / I.O. Tovakch, S.Y. Zhuk // IEEE First Ukraine Conference on Electrical and Computer Engineering, 1-2 June 2017, Kiev, Ukraine. DOI: 10.1109/UKRCON.2017.8100466.

75. Tovkach I.O. Adaptive filtration of parameters of the UAV movement based on the TDOAmeasurement sensor networks // I.O. Tovkach, S.Ya Zhuk // Journal of Aerospace Technology and Management. — 2019. — Vol. 11. — e3519. DOI: https://doi.org/10.5028/jatm.v11.1062.

76. Omelianenko M. E-plane stepped-impedance bandpass filter with wide stopband / M. Omelianenko, T. Romanenko // IEEE International Conference on Electronics and Nanotechnology, 22-24 April 2020, Kyiv, Ukraine. DOI: 10.1109/ELNANO50318.2020.9088888.

77. Omelianenko M.Yu. High efficiency waveguide-planar amplifier with spatial power combinig for frequency range 31-39 GHz. // M.Yu. Omelianenko, T.M. Romanenko // Radioelectronics and Communications Systems. - 2019. - Vol. 62. - P. 195-201. DOI: https://doi.org/10.3103/S0735272719050017.

78. Dubrovka F. Compact X-band stepped-thickness septum polarizer / F. Dubrovka, S. Piltyay, O. Sushko, R.Dubrovka, M. Lytvyn, S. Lytvyn // IEEE Ukraine Microwave Week, 21-25 Sept. 2020, Kharkiv, Ukraine. DOI: 10.1109/UkrMW49653.2020.9252583.

79.Liu Y. Design and optimization of wide and dual band waveguide polarizer / Y. Liu, F. Li, X. Li, H. He // Global Symposium on Millimeter Waves, Nanjing, China, April 2008. https://doi.org/10.1109/APMC.2000.925721.

80.Park B.M. Sensitivity analysis of square waveguide iris polarizers / B. M. Park, P. Ramunujam, F. Boldissar, C. Shin // Proceedings of IEEE Antennas and Propagation Society International Symposium and URSI National Radio Science Meeting, Seattle, USA, 1994, http://doi.org/10.1109/APS.1994.407908.

Фесюк Ірина Володимирівна - студентка групи РС-71, радіотехнічного факультету, Національний технічний університет України «Київський політехнічний інститут», Київ, e-mail: irinkafes@gmail.com;

Булашенко Андрій Васильович - ст. викл. кафедри теоретичних основ радіотехніки, Національний технічний університет України «Київський політехнічний інститут ім. Ігоря Сікорського», Київ.

Fesyuk Iryna $\boldsymbol{V}$. - Department of Radio Engineering, National Technical University of Ukraine «Igor Sikorsky Kyiv Polytechnic Institute», Kyiv, e-mail: irinkafes@gmail.com;

Bulashenko Andrew V. - Senior Lecturer of Department of Radio Engineering, National Technical University of Ukraine «Igor Sikorsky Kyiv Polytechnic Institute», Kyiv. 